\section{ScienceDirect}

www.sciencedirect.com
EM|consulte

www.em-consulte.com neuropsychiatrie

de l'enfance

et de l'adolescence

Neuropsychiatrie de l'enfance et de l'adolescence xxx (2017) xxx-xxx

\title{
Éditorial/Point de vue \\ Remarques épistémologiques et cliniques sur la notion de vulnérabilité psychotique
}

\author{
Epistemological and clinical considerations about the notion of psychotic vulnerability
}

\begin{abstract}
Résumé
L'article propose de soulever certaines questions cliniques et épistémologiques posées par la notion de vulnérabilité psychotique, dans le champ de la psychopathologie de l'enfant et de l'adolescent. La vulnérabilité s'est en effet constituée comme un paradigme majeur de la santé mentale contemporaine, suscitant un nombre considérable de recherches sur les facteurs de risque et d'apparition des troubles psychotiques. Les études consacrées au risque de psychose et à sa détection précoce ont marqué, depuis une vingtaine d'années, un tournant dans les modèles de vulnérabilité : les facteurs de vulnérabilité y sont englobés dans la catégorie dite à très haut risque (ultra-high risk), voire dans un syndrome de risque de psychose. Mais, concevoir la vulnérabilité comme risque pose plusieurs problèmes : celui d'une spécificité clinique suffisante pour fonder la prédiction, et celui de sa fidélité aux trajectoires développementales singulières. L'article examine ces interrogations à partir de deux exemples aujourd'hui paradigmatiques de la vulnérabilité psychotique : celui des expériences dites quasi-psychotiques chez l'adolescent, et celui du syndrome développemental de microdélétion 22q11.2.
\end{abstract}

(C) 2018 Elsevier Masson SAS. Tous droits réservés.

Mots clés : Vulnérabilité psychotique ; Syndrome de risque de psychose ; Expériences quasi psychotiques ; Syndrome de microdélétion 22q11.2 ; Clinique différentielle ; Angoisse ; Troubles anxieux ; Perte de la réalité ; Transition psychotique

\begin{abstract}
Background. - The goal of this paper is to shed light on specific clinical and epistemological questions raised by the notion of psychotic vulnerability in the field of child and adolescent psychiatry. This notion has nowadays become a major research paradigm and orients numerous studies on risk factors for transition to psychosis. In the last 20 years studies focusing on such risk factors and their early identification have given a new impetus to models of vulnerability: they unify vulnerability factors under the category of ultra-high risk, or even risk syndrome for psychosis.

Method. - A review of the conceptual and historical background framing the notion of vulnerability sheds light on epistemological and clinical ambiguities characterizing its scope and relevance for child and adolescent psychopathology. The paper aims to clarify these questions raised by the vulnerability models by focusing on two paradigm cases of vulnerability to psychosis: psychotic-like experiences in adolescents, and the $22 \mathrm{q} 11.2$ microdeletion developmental syndrome.

Results. - The discrepancy between the statistical background of vulnerability factors and a clinical understanding of the vulnerability involves two main difficulties for the clinical scope of these models: the first one relates to a specific vulnerability to psychosis, opposed to a general vulnerability to develop a mental disorder. The second one is the difficulty to grasp, from the perspective of risk models, the clinical and dynamic temporality of the transition toward psychotic symptoms.

Discussion. - These ambiguities raised by the ultra-high risk models call for a psycho-dynamic approach, regarding diagnosis in light of a subjective experience and trajectory, and of the psychic functions and dynamics of a symptom. Such a psycho-dynamic point of view can shed light on a clinical understanding of the vulnerability models.
\end{abstract}

(C) 2018 Elsevier Masson SAS. All rights reserved.

Keywords: Psychotic vulnerability; Ultra-high risk; Psychotic-like experiences; 22q11.2 microdeletion syndrome; Differential diagnosis; Anxiety disorders; Loss of reality; Psychotic transition 


\section{Introduction}

La recherche de marqueurs de vulnérabilité aux troubles mentaux, et en particulier à la schizophrénie ${ }^{1}$, constitue un paradigme qui ne cesse de croître dans les modèles théoriques et les dispositifs de soin en santé mentale. De très nombreuses études cliniques et épidémiologiques visent, depuis une vingtaine d'années, l'identification et la détection précoce de facteurs de risques associés à l'émergence de symptômes psychotiques [2]. Ce paradigme se situe au carrefour de plusieurs évolutions récentes en psychiatrie : d'une part, l'essor d'une approche dimensionnelle et des recherches sur les troubles de la personnalité - schizotypie, schizoïdie - qui formeraient une vulnérabilité psychotique ; d'autre part, l'essor des modèles génétiques et neuro-développementaux de la schizophrénie [3].

Or, la notion de vulnérabilité psychotique soulève des enjeux majeurs en psychiatrie de l'enfant et de l'adolescent, aussi bien épistémologiques que cliniques. Elle interroge l'articulation des niveaux d'explication, entre la clinique pédopsychiatrique et les modèles génétiques et neuro-développementaux. Elle redonne également une actualité aux problèmes soulevés par le diagnostic et la délimitation des entités cliniques chez l'enfant, telles qu'ils ont été discutés à propos de la psychose infantile [4], et de l'approche structurale en psychanalyse [5].

$\mathrm{Si}$ les recherches sur les marqueurs de vulnérabilité débouchent sur des modèles étiologiques de la schizophrénie, leur visée première, au plan clinique, est le développement de dispositifs de prévention et de prise en charge précoce des troubles psychotiques. La question de leur portée clinique est alors cruciale. Sachant que les études de vulnérabilité se fondent sur des corrélations statistiques, entre la présence de certains facteurs et l'apparition ultérieure d'une psychose, dans quelle mesure sont-elles à même d'éclairer l'émergence psychotique dans la trajectoire singulière d'un sujet? La notion de risque de psychose invite à prêter une attention spécifique au moment clinique de la transition entre des signes précurseurs et l'apparition des symptômes psychotiques : or, ce moment se caractérise généralement par une large part d'indétermination clinique. Il nous semble que les approches psychodynamiques, loin d'être disqualifiées par les modèles de vulnérabilité, s'avèrent alors particulièrement éclairantes pour discuter la pertinence de ces modèles, dans le repérage clinique de cette temporalité de l'émergence psychotique.

Après un bref aperçu historique sur la notion de vulnérabilité, nous tenterons d'expliciter les difficultés de ses implications cliniques, qui demeurent souvent implicites dans les travaux qui lui sont consacrés. Nous examinerons pour cela deux modèles majeurs de la vulnérabilité psychotique chez l'enfant et l'adolescent : celui des expériences dites quasi-psychotiques,

\footnotetext{
1 Nous utilisons le terme de schizophrénie dans le sens très large qui lui est donné dans les recherches contemporaines sur la vulnérabilité psychotique $[1,12]$. Pour cette raison, nous laissons de côté le débat - non tranché - sur la pertinence et l'extension de la catégorie de psychose appliquée à la clinique de l'enfant et de l'adolescent
}

et celui du risque de psychose dans une pathologie développementale d'origine génétique, la microdélétion 22q11.2.

\section{La vulnérabilité psychotique : arrière-plans historiques}

Les racines de la notion de vulnérabilité renvoient pour une large part, dans l'histoire de la psychiatrie, aux concepts de diathèse, de constitution et de prédisposition. Elle forme alors une jonction entre des facteurs endogènes héritables et des facteurs exogènes d'une pathologie. Pour la schizophrénie, Kretschmer [6] décrit en 1921 le type de caractère ou de personnalité schizoïde, repris par Bleuler [7] et Minkowski [8]. La schizoïdie est entendue comme une constitution qui témoigne d'un continuum entre normal et pathologique : «La plupart des symptômes schizophrènes manifestes apparaissent sur un mode psychique en fonction de certains fondements physiopathologiques. Il suffit alors, avec ces mécanismes très fins, d'une minuscule variation par rapport à la normalité pour faire naître les phénomènes psychiques de l'association dissociative, de l'hallucination, des idées délirantes etc. On voit même des esquisses de ce type de phénomène chez des sujets sains. » 7 p.46].

Les premiers modèles de la vulnérabilité à la schizophrénie, dans la seconde moitié du $20^{\mathrm{e}}$ siècle, accentuent ce rôle de jonction entre causes endogènes et exogènes, contribuant pour une grande part à l'essor des modèles poly-factoriels et bio-psychosociaux de la schizophrénie. Rado [9], forgeant la notion de schizotypie, de même que Meelh [10], qui théorise la schizotaxie, soulignent l'interaction entre des facteurs génétiques et neuro-cérébraux et les facteurs environnementaux. Les composantes de la personnalité ne deviennent symptômes de maladie que lorsqu'elles rencontrent un environnement défavorable, qui devient une cause déclenchante.

Le paradigme de la vulnérabilité psychotique se développe alors sur la base des modèles stress-vulnérabilité d'une part, et d'une approche dimensionnelle des troubles mentaux d'autre part [11]. Il s'agit notamment de déterminer des seuils de vulnérabilité qui correspondent au seuil de déclenchement de manifestations pathologiques lorsqu'un individu est soumis à un stress (biologique, psychologique ou social). Ces seuils de vulnérabilité, susceptibles de prendre place sur un continuum, s'appuient sur l'hypothèse de marqueurs génétiques et neurobiologiques.

Depuis une vingtaine d'années, les études sur la psychose débutante ont marqué un nouvel essor des modèles de vulnérabilité. Dans la lignée des travaux de l'école de Melbourne menée par McGorry, les recherches sur le ultra-high risk, appuyées sur l'hypothèse dimensionnelle d'un continuum psychotique, interrogent différents types de facteurs de vulnérabilité chez les adolescents : d'une part, la présence de symptômes psychotiques intermittents ou atténués (expériences quasi psychotiques) ; d'autre part, les troubles de la personnalité schizoïde ou schizotypique, fréquemment associés à la présence de sujets atteints de schizophrénie parmi les apparentés [12].

La littérature considérable suscitée par la notion de risque de psychose marque un tournant dans l'histoire de la vulnérabilité. Le concept de risque, dont la connotation est moins déterministe 
que le terme de vulnérabilité, place moins l'accent sur une prédisposition endogène - héritée de la psychiatrie constitutionnaliste - que sur la prévention et le case management, invitant à une attention particulière aux ressources de coping du sujet [13]. Les débats liés à l'entrée potentielle des entités de risque de psychose et de psychose atténuée dans le DSM5 illustrent la manière dont ces études informent aujourd'hui la conception de la maladie mentale elle-même [14] : les classifications diagnostiques n'opèreraient plus uniquement sur des pathologies, mais également sur le risque de pathologie.

Les études de risque de psychose s'appuient également sur l'essor de modèles génétiques et neuro-développementaux de la schizophrénie. Elles s'intéressent en particulier au risque accru d'apparition de troubles psychotiques dans certaines pathologies développementales. Dans la lignée des modèles de stressvulnérabilité, ces recherches posent la question des interactions entre les facteurs neurobiologiques et environnementaux, et de l'impact des altérations génétiques et neurodéveloppementales sur les registres psychologiques, relationnel et social de l'environnement des sujets.

\section{Du risque statistique au risque clinique ?}

En invitant à un déplacement de l'attention clinique, de la pathologie vers le risque de développer cette pathologie, la vulnérabilité et le risque sont susceptibles de transformer profondément la conception des troubles mentaux et la pratique diagnostique. Ils posent ainsi de manière cruciale la question éthique et institutionnelle des modalités et des dispositifs de prévention et de prise en charge précoce, adressés à des adolescents qui n'ont pas déclaré les symptômes avérés d'une psychose. La prise de traitements neuroleptiques à visée préventive, la stigmatisation induite par la catégorie de risque de psychose, les « faux positifs » découlant du hiatus entre réalité statistique et singularité clinique constituent autant d'enjeux éthico-cliniques à la portée considérable pour la santé mentale contemporaine.

Si l'apparition des premiers signes d'une psychose est une période le plus souvent marquée par une grande indétermination clinique, le moment de l'adolescence exige de redoubler de prudence quant à la pratique diagnostique. Dans la mesure où les entrées dans la psychose se situent le plus souvent à la fin de l'adolescence, la visée d'une détection précoce coïncide avec la période pubertaire, dans laquelle les manifestations symptomatiques peuvent être particulièrement intenses, tout autant que transitoires et mouvantes. La fragilité des assises narcissiques et identitaires dans ces moments de remaniements identificatoires est susceptibles de rendre d'autant plus aliénante, voire persécutante, l'assignation à une vulnérabilité psychotique, si cette dernière est posée comme une catégorie diagnostique essentialisante et déterministe.

Il nous semble qu'identifier et discuter précisément ces enjeux éthique et clinique invite tout d'abord à mieux cerner deux limitations qui caractérisent les modèles actuels de vulnérabilité : leur défaut de spécificité d'une part, et leur écart vis-vis d'une conception temporelle et dynamique de la transition psychotique d'autre part.

\subsection{Quelle spécificité clinique de la vulnérabilité ? Une clinique différentielle des marqueurs de risque}

Une première difficulté pour la pertinence clinique de la vulnérabilité est sa spécificité. La portée clinique et psychopathologique des facteurs de vulnérabilité est d'autant plus grande qu'ils correspondent à une pathologie spécifique. Or, de nombreux éléments des modèles de stress-vulnérabilité renverraient davantage à une vulnérabilité générale à développer un trouble psychique, qu'à une pathologie donnée. Au fil du développement de la psychiatrie génétique, il a été souligné que plutôt que de corroborer des associations binaires entre des génotypes et des phénotypes pathologiques, les marqueurs génétiques formeraient le plus souvent un risque général à développer un trouble mental, de nombreuses altérations génétiques paraissant transversales à différentes entités cliniques [15]. Il en va de même des composantes environnementales qui peuvent être désignées comme facteurs de stress : le rôle des événements traumatiques et de l'anxiété psychosociale est souligné comme déterminant dans de nombreuses pathologies (troubles bipolaires, troubles psychotiques, troubles anxieux et dépressifs), et se révèle par conséquent peu spécifique pour circonscrire précisément des facteurs différentiels de vulnérabilité [16].

Cette difficulté montre l'importance d'une clinique différentielle des signes précurseurs ou indicateurs d'un devenir pathologique [17]. Celle-ci s'avère particulièrement cruciale lorsqu'il s'agit d'explorer des signes qui seraient corrélés, chez l'enfant et l'adolescent, à une probabilité accrue de développer un trouble schizophrénique à l'âge adulte. Les modèles de vulnérabilité courent en effet le risque d'inventorier ces signes potentiels à partir du point de vue de la psychopathologie adulte, davantage que du point de vue développemental. Les indicateurs cliniques pensés à partir des formes adultes des troubles du spectre schizophréniques - symptômes positifs et négatifs -, tendent à occulter l'aspect polymorphe, mouvant et fluctuant que revêtent fréquemment les tableaux symptomatiques chez l'enfant. Ils sont également susceptibles de sous-estimer l'allure parfois psychotique, mais transitoire, qui peut caractériser certains moments de réorganisation au cours du développement, et les bouleversements psychiques engendrés par la puberté et le travail de l'adolescence. Les discussions portant, dans le champ de la pédopsychiatrie psychanalytique, sur la portée de la notion de structure dans la clinique de l'enfant et de l'adolescent [18] ont ainsi souligné la difficulté à effectuer une clinique différentielle des symptômes d'angoisse, des expériences de vacillement de la réalité ou encore des vécus hallucinatoires, sans négliger la dynamique développementale et l'indétermination clinique qui l'accompagne - difficulté d'autant plus grande que la palette des défenses et des expressions symptomatiques est particulièrement vaste chez l'enfant.

\subsection{De la vulnérabilité à la transition psychotique : du risque à l'émergence psychotique}

Si, en tant que donnée épidémiologique, la vulnérabilité est issue d'études de corrélations, l'émergence d'une psychose ne peut être saisie cliniquement qu'à partir de la temporalité 
d'une trajectoire clinique. Le moment du passage aux premiers symptômes d'une psychose interroge les rapports causaux et dynamiques entre signes précurseurs et symptômes avérés, les différentes formes que peut revêtir ce moment de transition, et les ressources subjectives qu'il laisse subsister. Ces questions requièrent de se placer dans la temporalité d'une trajectoire clinique : elles paraissent en ce sens laissées en suspens par les rapports de corrélation, qui risquent de faire de la vulnérabilité une catégorie figée, réduite à un inventaire de facteurs statiques.

Au sein de l'ensemble des signes susceptibles d'être corrélés à une vulnérabilité psychotique, un point de vue psychodynamique amène à explorer les vécus de l'émergence psychotique dans les différentes fonctions subjectives qu'ils peuvent revêtir - et qui peuvent témoigner de tentatives de lutte et de compensation vis-à-vis de cette émergence. Là aussi, l'importance de ce point de vue dynamique et temporel est particulièrement marquée dans la clinique de l'enfant et de l'adolescent. De nombreux travaux pointent, dans cette perspective, le risque inhérent à la notion de psychose infantile, de réifier et de figer la temporalité dynamique en une entité clinique statique. L'impact subjectif du diagnostic, tout autant que le recours, dans le développement, à des mécanismes défensifs d'allure psychotique mettent l'accent sur les difficultés que soulèverait un syndrome dit de risque de psychose, chez l'enfant et l'adolescent [19-21]. Les modèles de vulnérabilité doivent de ce point de vue être mis à l'épreuve, dans leurs implications psychopathologique et clinique, de descriptions subjectives du vécu des adolescents confrontés à ces moments de transition psychotique, et de modèles dynamiques de cette émergence symptomatique.

Deux domaines sont aujourd'hui au premier plan dans les études de vulnérabilité psychotique, et apparaissent particulièrement révélateurs de ces difficultés : celui des expériences dites de type psychotiques chez l'adolescent, et celui du syndrome neurodéveloppemental de microdélétion 22q11.2.

\section{Deux exemples cliniques du très haut risque de psychose}

\subsection{Les expériences dites quasi-psychotique chez l'adolescent}

Les études portant sur les expériences dites quasipsychotiques (psychotic like) ont joué un rôle décisif dans le développement d'une approche dimensionnelle des troubles psychotiques, et dans le renouvellement des modèles de vulnérabilité. Cette catégorie rend perceptibles plusieurs ambiguïtés cliniques et nosographiques. Les expériences quasipsychotiques regroupent notamment les formes ponctuelles et atténuées d'hallucinations, de croyances délirantes ou de vécu de perte de la réalité (déréalisation, dépersonnalisation), qui possèderaient une fréquence non négligeable au sein de la population générale (entre $10 \%$ et $20 \%$ ), et en particulier chez les adolescents (jusqu'à $20 \%$ ) [22]. Liées aux composantes schizoïde et schizotypique de la personnalité, elles seraient en faveur d'une conception continuiste de la schizophrénie, et formeraient un ensemble de facteurs de risque psychotique : «Les études que nous avons considérées indiquent que les hallucinations et les délires sont des phénomènes dimensionnels reposant sur des continuités avec les expériences normales. Bien que la grande majorité des individus éprouvant ces «symptômes » n’ont pas besoin de soins, les études longitudinales suggèrent qu'ils peuvent néanmoins présenter un risque augmenté de développer un trouble clinique ${ }^{2}$. $\gg[23$, p.1134].

Comme il a été souligné, cette catégorie très vaste, et dont la délimitation est plus statistique que clinique, met en relief la nécessité d'une clinique différentielle fine de ces expériences quasi psychotiques, sous peine que sa valeur prédictive ne soit amoindrie par de nombreux faux positifs, et sa portée psychopathologique limitée [24]. Cette catégorie risque en effet de sous-entendre que certaines expériences seraient en ellesmêmes de type psychotique, et que seules des différences de degré et d'intensité permettraient d'opérer des distinctions cliniques au sein des hallucinations ou des croyances inhabituelles. Or, à l'encontre de cette conception très extensive du qualitatif « psychotique », de nombreuses expériences transitoires de vacillement de la réalité, d'hallucinations ou de délires peuvent s'apparenter à des formes de dissociation névrotique, et témoigner d'un moment passager de désorganisation de l'économie psychique [25]. Le rôle des hallucinations ou des croyances dites « paranormales » comme mécanismes défensifs et tentatives de figuration ou de subjectivation a également été discuté dans la clinique du traumatisme [26]. La question de savoir dans quelle mesure ce type d'expériences forme un facteur de risque aux psychoses suppose ainsi d'interroger une typologie de ces vécus, et les différences qualitatives et subjectives qui peuvent les caractériser, selon les modalités de fonctionnement psychique du sujet, et les contextes cliniques dans lesquels ils surviennent.

Cette perspective suppose de ne pas s'en tenir à une qualification du symptôme, et de replacer le diagnostic différentiel au niveau d'une économie subjective globale et dynamique. $\mathrm{Si}$ certains signes cliniques, comme les hallucinations ponctuelles, sont statistiquement corrélés à un risque accru d'apparition d'une schizophrénie, la catégorie d'expériences quasi psychotiques tend à assimiler les signes psychotiques atténués, les marqueurs de vulnérabilité, et les prodromes d'un déclenchement psychotique. Or, du point de vue de la trajectoire et de l'évaluation clinique d'un sujet, ces trois catégories ne peuvent être confondues. D'une part, comme le souligne Marty en évoquant une « paranoïa ordinaire » de l'adolescent [27], des signes d'allure psychotique tels que des vécus persécutifs, ou un recours marqué aux mécanismes interprétatifs et projectifs, peuvent être à rapporter à la violence pubertaire, qui renforce notamment la fragilité narcissique, et ne doivent pas être d'emblée assimilés à des prodromes psychotiques [28]. D'autre part, des expériences de vacillement du rapport à la réalité comme la déréalisation et la dépersonnalisation, qui ne sont pas rares, elles aussi, chez l'adolescent, peuvent parfois être entendues comme une lutte défensive contre une dislocation psychique et une perte de la réalité psychotiques, davantage que comme des marqueurs d'une psychose débutante ou à venir [29,30]. Au sein des facteurs de vulnérabilité, l'évaluation et la

\footnotetext{
2 Il s'agit de notre traduction.
} 
prise en charge précoces des adolescents dits à risque de psychose questionnent les fonctions de défense ou les possibilités de suppléance dont peuvent témoigner certains symptômes, face à la menace d'une transition vers la schizophrénie.

\subsection{Le syndrome développemental de microdélétion}

$22 q 11.2$

Le syndrome de microdélétion 22q11.2 est une pathologie développementale, regroupant notamment le syndrome de DiGeorge et le syndrome vélocardiofacial ; elle est sous-tendue par une altération génétique commune (une microdélétion, c'està-dire une perte, d'une partie du bras long du chromosome 22). Ce syndrome a suscité récemment des travaux nombreux, à l'intersection de la psychiatrie génétique et de la clinique pédopsychiatrique. Le risque de psychose y tient en effet une place prépondérante : l'apparition d'une schizophrénie concernerait environ $30 \%$ de ces patients [31]. Ce syndrome apparaît ainsi comme « un facteur de risque génétique hautement pertinent pour la schizophrénie et comme le modèle humain le plus prometteur pour l'étude des facteurs de risque et des états à risque pour la schizophrénie ${ }^{3}$. » $[1, p .259]$. Il constitue en ce sens le paradigme d'un modèle intégratif de la vulnérabilité, qui associerait les signes cliniques précurseurs d'une psychose à de potentiels marqueurs neurocognitifs et génétiques [32].

La microdélétion 22q11.2 est associée à un large spectre de manifestations somatiques, neurocognitives et psychopathologiques. Les anomalies développementales organiques touchent notamment le cœur, les systèmes immunitaire et endocrine, ainsi que la face, entrânant en particulier des troubles de la déglutition et de l'articulation. Les difficultés psychomotrices dans les apprentissages s'associent fréquemment à un retard du langage et à une déficience intellectuelle modérée, ainsi qu'à des troubles de l'attention et de la cognition sociale [33]. La comorbidité avec des troubles psychopathologiques est conséquente, et concerne notamment les troubles de l'attention et les troubles anxieux chez l'enfant, et les troubles psychotiques et les troubles de l'humeur chez l'adolescent et l'adulte. Or, les études longitudinales sur de potentiels marqueurs du risque psychotique accordent une place prépondérante à la présence de troubles anxieux dans les trajectoires développementales de ces patients [34]. La microdélétion 22q11.2 viendrait alors confirmer des résultats ayant déjà souligné, au sein de la population générale, la présence d'un risque accru de schizophrénie chez les sujets présentant des troubles anxieux [35].

Ce lien entre anxiété et risque de psychose illustre plusieurs questions soulevées par la portée des modèles de vulnérabilité pour la clinique différentielle et la transition psychotique. Tout d'abord, sous quel aspect l'anxiété peut-elle être indicatrice d'une vulnérabilité spécifique aux troubles psychotiques? Cela implique qu'une forme spécifique d'anxiété soit distinguée du rôle global qui reviendrait au stress dans une vulnérabilité générale aux troubles mentaux, via ses répercutions sur le développement affectif et cognitif, sur le fonctionnement

3 Il s'agit de notre traduction. neurobiologique et cérébral, et sur la capacité d'ajustement à l'environnement [36]. On retrouve là la question de la clinique différentielle, et des différents types d'anxiété, pour éclairer le lien particulier entre troubles anxieux et risque de psychose chez les enfants et adolescents atteints de ce syndrome. Les divers troubles anxieux répertoriés dans les classifications psychiatriques internationales semblent se rencontrer chez ces enfants : phobies spécifiques, phobie sociale, trouble anxieux généralisé, anxiété de séparation, et troubles obsessionnels-compulsifs. On peut alors se demander si, au-delà d'un tel inventaire symptomatique, une typologie de l'angoisse, telle qu'elle se rencontre au sein de la psychopathologie psychanalytique, pourrait contribuer à éclairer cette vulnérabilité spécifique aux psychoses.

Cette perspective est, là aussi, indissociable d'un questionnement sur les rapports dynamiques et causaux qui pourraient rendre compte du passage, sur le plan psychique et subjectif, entre les symptômes anxieux et l'apparition d'une symptomatologie psychotique. Les difficultés somatiques et neurocognitives vécues par ces enfants dès les phases précoces du développement, associées aux répercussions du handicap et des interventions médicales sur l'économie psychique et le fonctionnement familial, questionnent la manière dont sont traversées ces phases marquées par les angoisses de démantèlement, d'anéantissement, d'indifférenciation, puis d'abandon et de séparation. Une hypothèse serait notamment de relier, chez ces enfants, la vulnérabilité psychotique à une prédominance de formes archaïques d'angoisses et de défenses, qui pourraient se révéler plus spécifiques que la symptomatologie des troubles anxieux. Les symptômes psychotiques, dont il n'est pas rare qu'ils surgissent dès l'enfance chez ces patients [37], pourraient, dans ce contexte clinique, être interrogés comme des défenses massives contre la résurgence d'éléments précoces à résonance traumatique, ou non métabolisés, suscités par les atteintes somatiques et les souffrances du handicap [38].

Les corrélations entre anxiété et devenir psychotique questionnent également la fonction défensive que peuvent revêtir certains symptômes anxieux face aux angoisses archaïques et au risque d'une émergence psychotique. L'évitement phobique, aussi bien que les comportements obsessionnels-compulsifs, pourraient marquer, dans cette mesure, un indicateur du risque de psychose, en tant qu'ils témoigneraient d'une tentative de circonscrire, de canaliser ou d'externaliser des éléments difficilement élaborables, et susceptibles de faire surgir des défenses ou des expériences de perte de la réalité de type psychotique. La question du rôle de l'anxiété dans ce modèle de la vulnérabilité schizophrénique interroge particulièrement l'enchaînement temporel et les relations psychodynamiques des différents signes cliniques qui peuvent marquer la transition vers une psychose, et qui ne peuvent être abordés qu'au sein de la trajectoire développementale et du contexte clinique propre à un sujet.

\section{Conclusion}

Si les études de vulnérabilité constituent aujourd'hui un paradigme central dans l'épistémologie et les recherches cliniques 
en psychopathologie, celles-ci ne forment pas pour autant un champ unifié et homogène. Les recherches autour des expériences quasi-psychotiques - représentatives d'une nouvelle approche dimensionnelle de la vulnérabilité psychotique - et celles qui portent sur le syndrome de microdélétion $22 q 11.2$ - emblématiques de l'essor des modèles développementaux et neuro-génétiques -, révèlent toutes deux les ambiguïtés cliniques et épistémologiques de l'identification du risque de psychose. Nous avons ici proposé de discuter deux difficultés principales que les modèles de vulnérabilité adressent à la clinique de l'enfant et de l'adolescent : leur spécificité clinique, et leur capacité à saisir la temporalité et la dynamique de la transition psychotique. Les enjeux éthiques et cliniques qui en découlent appellent une approche psychodynamique et différentielle, susceptible de jalonner le passage d'une vulnérabilité statistique à une vulnérabilité clinique, cherchant à saisir la temporalité et la subjectivité d'une entrée dans la schizophrénie.

\section{Déclaration de liens d'intérêts}

Les auteurs déclarent ne pas avoir de liens d'intérêts.

\section{Références}

[1] Schneider M, Armando A, Pontillo M, Vicari S, Debbané M, Schultze-Lutter F, et al. Ultra high risk status and transition to psychosis in 22q11.2 deletion syndrome. World Psychiatry 2016;15(3): 259-65.

[2] Fusar-Poli P, Borgwardt S, Bechdolf A, Addington J, RiecherRössler A, Schultze-Lutter F, et al. The psychosis high-risk state: a comprehensive state-of-the-art review. JAMA psychiatry 2013;70(1): 107-20.

[3] Bonnot $\mathrm{O}$, Mazet $\mathrm{P}$. Vulnérabilité aux schizophrénies à l'adolescence : revue de la littérature et applications cliniques. Neuropsychiatr Enfance Adolesc 2006;54:92-100.

[4] Speranza M. Approche psychopathologique et développementale de la schizophrénie infantile. Neuropsychiatr Enfance Adolesc 2006;54: $45-53$.

[5] Misès R. L'influence des conceptions dynamiques sur l'organisation de la pédopsychiatrie en France. Ann Med Psychol 2001;159(3): $173-6$.

[6] Kretschmer E. La structure du corps et le caractère. Paris: Payot; 1921. p. 1930.

[7] Bleuler E. Les problèmes de la schizoïdie et de la synthonie. Info Psychiatr 2011;87:37-51

[8] Minkowski E. La schizophrénie. Paris: Payot; 1927. p. 2002.

[9] Rado S. Dynamics and classification of disordered behaviour. Am J Psychiatry 1953;110:406-16.

[10] Meehl PE. Schizotaxia, schizotypy, schizophrenia. Am Psychol 1962;17(12):827-38.

[11] Zubin J, et Spring B. Vulnerability - a new view of schizophrenia? J Abnorm Psychol 1977;86(2):103-26.

[12] Yung AR, Phillips LJ, Yuen HP, McGorry PD. Risk factors for psychosis in an ultra high-risk group: psychopathology and clinical features. Schizophr Res 2004;67(2):131-42.

[13] Marion-Veyron R, Mebdouhi N, Baumann PS, Thonney J, Crespi S, et Conus P. Les premiers épisodes psychotiques : de l'importance du case management. Evol Psychiatr 2013;78:41-51.

[14] DSM-5 Task Force. Proposed draft revisions to DSM disorders and criteria: attenuated psychotic symptoms syndrome. Am Psychiatr Assoc 2010.

[15] Kendler KS. "A gene for": the nature of gene action in psychiatric disorders. Am J Psychiatry 2005;162(7):1243-52.
[16] Elzinga BM, Roelofs K, Tollenaar MS, Bakvis P, van Pelt J, Spinhoven P. Diminished cortisol responses to psychosocial stress associated with lifetime adverse events: a study among healthy young subjects. Psychoneuroendocrinology 2008;33(2):227-37.

[17] Sass LA. Self-disturbance and schizophrenia: structure, specificity, pathogenesis. Res Psychoanal 2013;16:119-32.

[18] Golse B. Structure des états ou structure des processus? Carnet Psy 2004;7:26-32.

[19] Diatkine R. Du normal et du pathologique dans l'évolution mentale de l'enfant (ou de limites de la psychiatrie infantile). Psychiatr Enfant 1967;10(1):2-42.

[20] Mazet P, Lebovici S. Autisme et psychoses de l'enfant. Paris: PUF; 1990.

[21] Askenazy F, Dupuis G, Dor E, Lestideau K, Meynadier A, Myquel M. Clinique des hallucinations auditives chez l'enfant non psychotique. Neuropsychiatr Enfance Adolesc 2009;57(1):25-31.

[22] Verdoux H, et van Os J. Psychotic symptoms in non-clinical populations and the continuum of psychosis. Schizophr Res 2002;54: $59-65$.

[23] Johns LC, van Os J. The continuity of psychotic experiences in the general population. Clin Psychol Rev 2001;21(8):1125-41.

[24] Evrard R. Les expériences réputées psychotiques dans la population générale : essai de problématisation. Ann Med Psychol Rev Psychiatr 2011;169(5):282-7.

[25] Maleval JC. Folies hystériques et psychoses dissociatives. Paris: Payot; 2007.

[26] Rabeyron T, Chouvier B, Le Maléfan P. Clinique des expériences exceptionnelles: du trauma à la solution paranormale. Evol Psychiatr 2010;75(4):633-53.

[27] Marty F. La violence comme expression du mal-être à l'adolescence. Adolescence 2009;4(70):1007-17.

[28] Le Maléfan P. "La vérité est ailleurs » La place du paranormal comme mode de traitement du réel pubertaire. Adolescence 2008;3(65): $709-21$.

[29] De Luca M. La dépersonnalisation à l'adolescence: entre étrangeté ordinaire et conscience disloquée. Evol Psychiatr 2015;80: $71-82$.

[30] Troubé S. La dépersonnalisation : l'ambiguïté clinique d'une notion transnosographique. Evol Psychiatr 2017;82/4:817-28 [In press].

[31] Schneider M, Debbané M, Bassett AS, Chow EW, Fung WLA, van den Bree MB, et al. Psychiatric disorders from childhood to adulthood in 22q11.2 deletion syndrome: results from the International Consortium on Brain and Behavior in 22q11.2 Deletion Syndrome. Am J Psychiatry 2014;171(6):627-39

[32] Stephenson DD, Beaton EA, Weems CF, Angkustsiri K, Simon TJ Identifying patterns of anxiety and depression in children with chromosome 22q11.2 deletion syndrome: comorbidity predicts behavioral difficulties and impaired functional communications. Behav Brain Res 2015;276:190-8.

[33] Swillen A, McDonald-McGinn. D. Developmental trajectories in 22q11.2 deletion syndrome. Am J Med Genet C 2015;169(2): $172-81$.

[34] Gothelf D, Schneider M, Green T, Debbané M, Frisch A, Glaser B, et al Risk factors and the evolution of psychosis in 22q11.2 deletion syndrome: a longitudinal 2-site study. J Am Acad Child Adolesc Psychiatry 2013;52(11):1192-203.

[35] Tien AY, Eaton WW. Psychopathologic precursors and sociodemographic risk factors for the schizophrenia syndrome. Arch Gen Psychiatry 1992;49(1):37-46.

[36] Pine DS. Integrating research on development and fear learning: a vision for clinical neuroscience? Depress Anxiety 2009;26(9):775-9.

[37] Debbané M, Glaser B, David MK, Feinstein C, Eliez S. Psychotic symptoms in children and adolescents with 22q11.2 deletion syndrome: neuropsychological and behavioral implications. Schizophr Res 2006;84(2):187-93.

[38] Potier R, Putois O, Lyonnet S, Weitzman JB, Villa F. Regards croisés sur une pathologie développementale d'origine génétique. Réflexions épistémologiques et cliniques sur l'impact psychique du syndrome de microdélétion 22q11.2. Topique 2016;135:103-17. 
S. Troubé a,* $^{*}$

R. Potier ${ }^{b}$

${ }^{a}$ Université de Nice, Sophia-Antipolis, LIRCES, 98, boulevard Edouard-Herriot, 06200 Nice, France

${ }^{\mathrm{b}}$ Campus Paris Rive-Gauche, centre de recherches psychanalyse, médecine et Société, EA3522, université Paris Diderot, Sorbonne Paris Cité, 5, rue Thomas-Mann, 75205 Paris cedex 13, France
O. Putois Université de Strasbourg, SuLiSom EA3071, 12, rue Goethe, 67000 Strasbourg, France

* Auteur correspondant. Adresses e-mail : sarah.troube@gmail.com (S. Troubé), r.potier@me.com (R. Potier), olivier.putois@gmail.com

(O. Putois)

Pour citer cet article : Troubé S, et al. Remarques épistémologiques et cliniques sur la notion de vulnérabilité psychotique. Neuropsychiatr Enfance Adolesc (2017), https://doi.org/10.1016/j.neurenf.2018.02.001 